\title{
Clinical Evaluation of Free-Breathing Contrast-Enhanced T1w MRI of the Liver using Pseudo Golden Angle Radial k-Space Sampling Klinische Evaluation der kontrastmittelverstärkten Leber-MRT bei freier Atmung mittels radialer „Pseudo-Golden-Angle“-k-Raum- Abtastung
}

Authors

Dennis M. Hedderich ${ }^{1,2}$, Kilian Weiss ${ }^{3}$, Judith E. Spiro ${ }^{1,4}$, Daniel Giese ${ }^{1}$, Gabriele M. Beck ${ }^{5}$, David Maintz ${ }^{1}$, Thorsten Persigehl ${ }^{1}$

Affiliations

1 Department of Radiology, University Hospital Cologne, Germany

2 Department of Diagnostic and Interventional Neuroradiology, Klinikum rechts der Isar, Technical University Munich, Munich, Germany

3 Philips Healthcare, Hamburg, Germany

4 Department of Radiology, University Hospital, LMU Munich

5 Philips Healthcare, Best, The Netherlands

Key words

MR-imaging, liver, free-breathing, radial, pseudo golden angle

received 16.08 .2017

accepted 10.01.2018

Bibliography

DOI https://doi.org/10.1055/s-0044-101263

Published online: 13.3.2018

Fortschr Röntgenstr 2018; 190: 601-609

(c) Georg Thieme Verlag KG, Stuttgart · New York

ISSN 1438-9029

Correspondence

PD Dr. Thorsten Persigehl

Department of Radiology, University Hospital Cologne,

Kerpener Str. 62, 50937 Cologne, Germany

Tel.: ++49/2 21/47882035

Tel.: ++49/221/4786787

thorsten.persigehl@uk-koeln.de

\section{ZUSAMMENFASSUNC}

Ziel Für die kontrastmittelverstärkte Magnetresonanztomografie (MRT) der Leber wird üblicherweise eine T1w-FS-FFE in Atemanhaltetechnik durchgeführt, um Bewegungsartefakte zu minimieren und eine gute diagnostische Qualität zu gewährleisten. Allerdings kann eine insuffiziente Durchführung des Atemanhaltemanövers die Bildqualität deutlich negativ beeinflussen. Ziel dieser Arbeit war es, die Bildqualität einer neuartigen MR-Sequenz mit radialer „Pseudo-Golden-Angle“-
k-Raumabtastung in freier Atmung in der klinischen Routine zu evaluieren.

Material und Methoden Es erfolgte eine retrospektive Auswertung der Leber-MRT in der späten Kontrastmittelphase bei 86 Patienten, die sowohl mittels der konventionellen kartesianischen k-Raumabtastung (Cartesian-eTHRIVE) in Atemanhaltung $(\mathrm{BH})$ als auch mittels der radialen „Pseudo-GoldenAngle“-k-Raumabtastung (Radial-eTHRIVE) in freier Atmung (FB) untersucht wurden. Es wurden sowohl objektive als auch subjektive Qualitätsparameter durch zwei verblindete Reader erhoben und mittels Wilcoxon-Test und gepaartem t-Test verglichen $(p<0,05)$.

Ergebnisse Die Untersuchungszeit war für die FB-RadialeTHRIVE signifikant länger als für die BH-Cartesian-eTHRIVE ( 2 min 54 s vs. 0 min 15 s). Subjektiv zeigte sich ein besseres Kontrastverhalten der Cartesian-eTHRIVE mit einem objektiv höheren Kontrast für hypointense Leberläsionen $(0,33 \pm 0,19$ vs. $0,20 \pm 0,11 ; p=0,000)$. Die subjektive Bewertung ergab eine signifikante Überlegenheit bezügliche der Schärfe der Anatomie und der Gefäße der FB-Radial-eTHRIVE. Im Falle starker Atemartefakte in der BH-Cartesian-eTHRIVE zeigte sich in freier Atmung mit der Radial-eTHRIVE weiterhin eine gute diagnostische Bildqualität.

Schlussfolgerung Die radiale Pseudo-Golden-Angle-T1w-FSFFE liefert eine hohe diagnostische Bildqualität in der kontrastmittelverstärkten Leber-MRT bei freier Atmung, sodass die Radial-eTHRIVE in freier Atmung bei Patienten mit ausgeprägten Atemartefakten in der DCE-MRT eine sehr hilfreiche „add-on“-Sequenz in der klinischen Routine darstellt.

\section{Kernaussagen}

- Die radiale Pseudo-Golden-Angle-k-Raumabtastung in der späten KM-Phase der Leber-MRT liefert zuverlässig eine gute diagnostische Bildqualität.

- Im Falle starker Atemartefakte in der konventionellen k-Raumabtastung bleibt eine hohe diagnostische Bildqualität erhalten.

- Der Kontrast hypointenser Leberläsionen ist für die RadialeTHRIVE herabgesetzt. 


\section{ABSTRACT}

Purpose Contrast-enhanced T1-weighted MR imaging of the liver is typically acquired using breath-hold techniques to reduce motion artifacts and to allow for optimal diagnostic image quality. Insufficient breath-holds during MR data collection can cause severe reduction of image quality up to the point of being non-diagnostic. The aim of this study was to evaluate the subjective and objective clinical image quality of a novel free-breathing radial k-space sampling MR technique.

Materials and Methods Consent for this study was given by the local IRB committee. 86 patients who underwent both breath-hold (BH) and free-breathing (FB) late-phase contrast T1w-FS-FFE liver MRI using conventional BH Cartesian (Cartesian-eTHRIVE) and FB "pseudo golden angle" radial k-space sampling (Radial-eTHRIVE) were included in this retrospective analysis. Subjective analysis comprised 5-point Likert scale ratings ( 1 = very good; 5 = non-diagnostic) for "artifact impact”, "anatomic sharpness", "vessel sharpness", "contrast impression", and "overall diagnostic quality". Relative signal intensities in different ROls were compared between Cartesian-eTHRIVE and Radial-eTHRIVE. For statistical differences paired Wilcoxon test and paired t-test have been performed $(\mathrm{p}<0.05)$.

Results The MR scan time was significantly longer for FB Radial-eTHRIVE (2 min, 54 s) compared to BH Cartesian-eTHRIVE (0 min 15 s). Cartesian-eTHRIVE demonstrated a superior subjective contrast impression and objective measurements revealed an increased lesion-to-liver-contrast for hypointense liver lesions (Hypo-LTLC: $0.33 \pm 0.19$ vs. $0.20 \pm 0.11$; $p=0.000$ ), while no difference was observed for hyperintense liver lesions (Hyper-LTLC). Subjective evaluation showed superior anatomic sharpness ratings by both readers for Radial-eTHRIVE. Most importantly, in a subgroup analysis of patients who were unable to perform adequate breath-holds, free-breathing Radial-eTHRIVE still demonstrated good subjective image quality.

Conclusion Free-breathing, radial k-space sampling T1w MRI of the liver delivers high diagnostic image quality, especially in patients who are unable to adequately perform breath-hold maneuvers. Thus, Radial-eTHRIVE can be an important clinical alternative in patients with impaired respiration status.

\section{Key points}

- Delayed-phase contrast-enhanced MRI of the liver can be robustly performed using a "pseudo golden angle" RadialeTHRIVE sequence.

- Free-breathing Radial-eTHRIVE yields good diagnostic image quality in case of a high artifact burden in breath-hold Cartesian-eTHRIVE and thus could be used as a "back-up" for patients with impaired respiratory capacity.

- A lower lesion-to-liver-contrast ratio is observed for hypointense liver lesions in free-breathing Radial-eTHRIVE sequence.

\section{Citation Format}

- Hedderich DM, Weiss K, Spiro JE et al. Clinical Evaluation of Free-Breathing Contrast-Enhanced T1w MRI of the Liver using Pseudo Golden Angle Radial k-Space Sampling. Fortschr Röntgenstr 2018; 190: 601-609

\section{Introduction}

Magnetic resonance imaging (MRI) is a clinical mainstay in the detection and characterization of both focal liver lesions and diffuse liver disease [1 -4]. In particular, contrast-enhanced T1-weighted sequences (CE- T1w) provide crucial information for the differential diagnosis of focal liver lesions (FLL) [5]. State-of-the-art MR protocols of the liver comprise 3 D T1-w fat-saturated fast-fieldecho sequences (T1w-FS-FFE) acquired dynamically before and after the administration of intravenous extracellular or hepatocyte-specific contrast agents. These T1w-FS-FFE sequences with Cartesian k-space sampling have different vendor-specific names, such as eTHRIVE ("enhanced T1 high-resolution isotropic volume excitation"; Philips), VIBE ("volumetric interpolated breath-hold examination”; Siemens) or LAVA ("Liver Acquisition with Volume Acceleration“; GE) [6, 7]. Dynamic contrast-enhanced (DCE) imaging with consecutive breath-holds allows for the acquisition of pre-contrast, arterial-phase, portal-venous phase, equilibrium, and delayed-phase images using bolus triggering or fixed timepoint methods [8]. However, the diagnostic imaging quality could be dramatically hampered by breathing artifacts in conventional breath-hold techniques with necessary consecutive breath-holds of about 15-20 seconds depending on coverage and resolution. Especially in the case of patients with compromised respiratory function, elderly patients, and children who may not be able to adequately hold their breath, impairment of image quality and subsequent diagnostic inaccuracy can be a severe clinical issue [9].

Different MR approaches using respiratory triggering and gating utilizing data from specific motion states have been developed to reduce respiratory-induced motion artifacts [10 - 12]. Moreover, k-space undersampling techniques and parallel imaging have led to a significant acceleration of $3 \mathrm{D}$ T1w-FS sequences with acquisition times of $<10$ seconds per dynamic scan $[13,14]$. On the other hand, novel radial k-space sampling techniques acquired during free breathing have been shown to be robust against respiratory motion-induced artifacts [15-17].

The purpose of this retrospective study was to evaluate the subjective and objective clinical image quality of a novel 3 D T1wFS FFE sequence based on "pseudo golden angle" radial k-space sampling (Radial-eTHRIVE). Therefore free-breathing RadialeTHRIVE images of the delayed post-contrast phase were compared to the standard breath-hold 3 D T1w-FS FFE with Cartesian k-space sampling (Cartesian-eTHRIVE). 
- Table 1 Imaging parameters for Cartesian-eTHRIVE and Radial-eTHRIVE.

- Tab. 1 Technische Parameter für Cartesian-eTHRIVE und Radial-eTHRIVE.

\begin{tabular}{|l|l|l|}
\hline & Cartesian-eTHRIVE & Radial-eTHRIVE \\
\hline field of view & $375 \times 352 \times 238 \mathrm{~mm}^{3}$ & $450 \times 450 \times 238 \mathrm{~mm}^{3}$ \\
\hline resolution & $1.4 \times 2.1 \times 4 \mathrm{~mm}^{3}$ & $1.5 \times 1.5 \times 4 \mathrm{~mm}^{3}$ \\
\hline sense factor & 2 & 1.4 \\
\hline partial fourier & $0.7(\mathrm{y}), 0.8(\mathrm{z})$ & $0.7(\mathrm{z})$ \\
\hline repetition time & $3.0 \mathrm{~ms}$ & $3.8 \mathrm{~ms}$ \\
\hline echo time & $1.47 \mathrm{~ms}$ & $1.55 \mathrm{~ms}$ \\
\hline scan time & $0 \mathrm{~min} 15 \mathrm{~s}$ & $2 \mathrm{~min} 54 \mathrm{~s}$ \\
\hline
\end{tabular}

\section{Methods}

\section{Study population and patient characteristics}

A total of $n=86$ adult patients who were referred to our Department of Radiology for diagnostic MR imaging of the liver were retrospectively evaluated in this study. The inclusion criteria were: patient age $\geq 18$ and patients who underwent Cartesian-eTHRIVE and Radial-eTHRIVE in the delayed contrast phase of the liver after intravenous application of the extracellular Gd-based contrast agent Gd-DOTA ( 0.1 mmol/kg body weight; Dotarem ${ }^{\circledR}$; Guerbet). The exclusion criteria were: MR examination at a different field strength or system (e.g. 1.5 T), or the use of a different contrast agent (e. g. Gd-EOB-DTPA). Consent for this study was given by the local IRB committee.

\section{MR imaging}

All MR examinations were performed on a 3.0 T whole-body MR system (Ingenia 3.0T; Philips Healthcare, Best, The Netherlands) using a 28-channel phased array coil.

All 86 patients underwent dynamic contrast-enhanced (DCE-) MR imaging of the liver including breath-hold Cartesian-eTHRIVE with an acquisition time of 0 min $15 \mathrm{~s}$ and free-breathing RadialeTHRIVE with an acquisition time of 2 min 54 s during the delayed contrast phase. Breath-hold Cartesian-eTHRIVE and free-breathing Radial-eTHRIVE were acquired 10 min following contrast administration in a clinically routine randomized order. Field-ofview dimensions were adjusted for the Radial-eTHRIVE sequence in order to avoid foldover artifacts. Before image acquisition of the free-breathing Radial-eTHRIVE sequence, patients were asked to maintain shallow and regular respiration. Detailed sequence parameters are given in $>$ Table 1.

The free-breathing Radial-eTHRIVE sequence consisted of a radial k-space sampling technique with a "pseudo golden angle" increment in the axial plane and Cartesian encoding in the z-direction, forming a so-called stack of stars sampling scheme. Each angle increment was based on the golden angle of $111.246^{\circ}$ and rounded such that each radial spoke falls onto the consecutive position of the corresponding evenly distributed radial scheme. This leads to an evenly distributed k-space, as illustrated in $\mathbf{F i g . 1}$. In addition, to reduce the number of encoding steps in the z-direction, parallel imaging (SENSE) and partial Fourier sampling were used.

\section{Image analysis}

A total of 172 delayed phase MR sequences of the 86 patients with Radial-eTHRIVE $(n=86)$ and Cartesian-eTHRIVE $(n=86)$ were subjectively analyzed by two radiologists with abdominal imaging experience of more than 3 years and 10 years (DMH and TP), respectively. The scans were presented in a random order and the radiologists were blinded to sequence names and specifications.

Subjective image quality was independently assessed using a 5-point Likert scale for the following categories based on previous evaluations $[18,19]$ ( $\triangleright$ Table 2): artifact impact (1: not present; 2: low impact; 3: moderate impact, 4: strong impact, 5: non-diagnostic), anatomic sharpness (1: very good; 2: good; 3: fair; 4: poor; 5: non-diagnostic), vessel sharpness (1: very good; 2: good; 3: fair; 4: poor; 5: non-diagnostic), contrast impression (1: very good; 2: good; 3: fair; 4: poor; 5: non-diagnostic), and overall diagnostic quality (1: very good; 2 : good; 3 : fair; 4: poor; 5: nondiagnostic). For subgroup evaluation of patients who were unable to hold their breath at conventional breath-hold Cartesian-eTHRIVE during the delayed phase, breathing artifact scores of 3 or higher - representing a moderate to non-diagnostic artifact impact - have been defined.

Objective measurements included signal intensities of the liver parenchyma (Liver-SI; the mean value of three regions-of-interest (ROI), two placed in the right hepatic lobe, one in the left hepatic lobe), of the autochthonic paraspinal muscle (Muscle-SI; single ROI in an area without obvious artifacts) and of the portal vein (PVSI; single ROI in an area without obvious artifacts). In the case of a present focal liver lesion (FLL), the signal intensity was measured in ROls of hypointense and hyperintense liver lesions (Hypo-LL and Hyper-LL, respectively).

Based on these ROI measurements, the relative liver-signal-intensity ratio (RLSI), liver-to-vessel contrast ratio (LTVC), hypointense lesion-to-liver contrast ratio (Hypo-LTLC) and hyperintense lesion-to-liver contrast ratio (Hyper-LTLC) were calculated in line with previous studies [20]: 


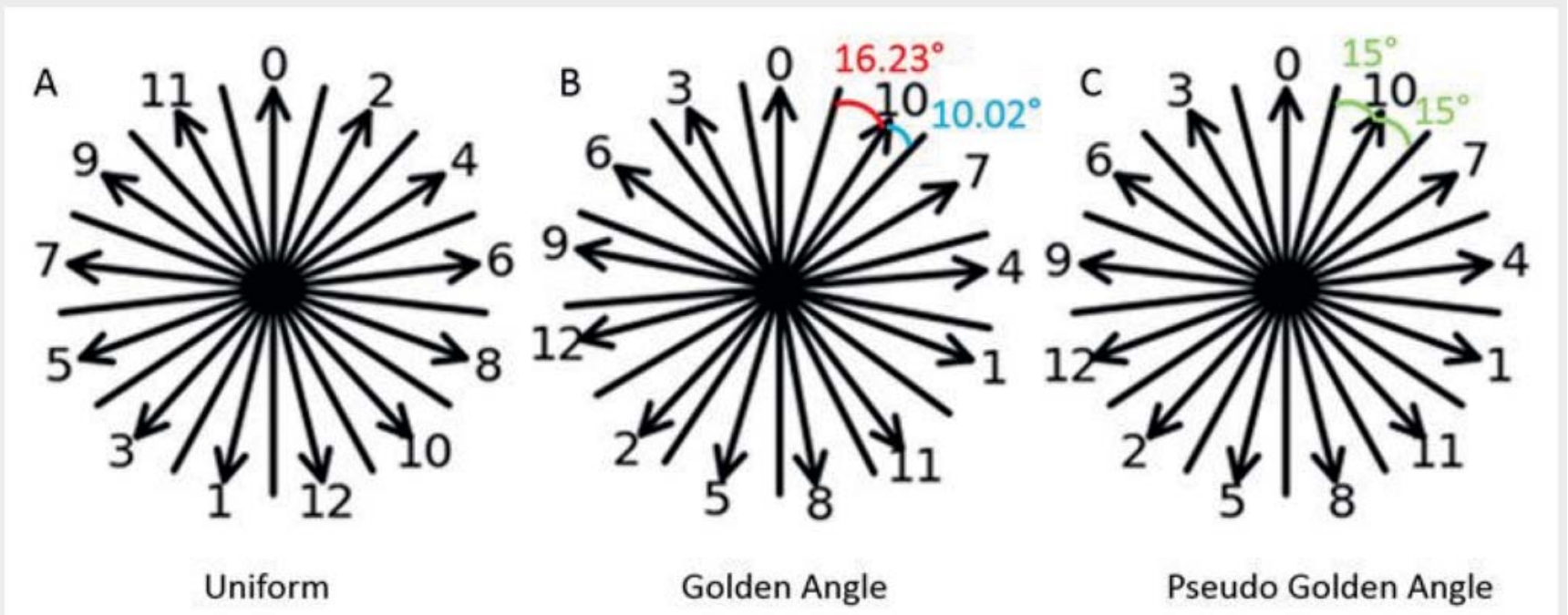

- Fig. 1 Uniform A, golden angle $\mathbf{B}$ and pseudo golden angle $\mathbf{C}$ k-space sampling schemes for radial imaging. In contrast to uniform sampling, the angle increment for pseudo golden angle sampling is $111.246^{\circ}$, leading to a more homogeneous sampling in space and time. However, golden angle sampling leads to non-equidistantly spaced radial spokes. This is overcome by pseudo golden angle sampling where the angle of every individual spoke is rounded towards the next position of a uniform sampling scheme.

- Abb. 1 A zeigt eine uniforme, B eine Abtastung mit „Golden-Angle“ und C eine Abtastung mit „Pseudo-Golden-Angle“. Die Pseudo-GoldenAngle-Abtastung benutzt einen Winkel von $111,246^{\circ}$ und sorgt somit für eine zeitlich und örtlich homogenere k-Raum-Füllung im Vergleich zur uniformen Abtastung oder zur Golden-Angle-Abtastung.

- RLSI $=1+$ (Liver SI - Muscle SI) / (Liver SI + Muscle SI)

- LTVC = (PVSI - Liver SI) / (PVSI + Liver SI)

- Hypo-LTLC = (Liver SI - Hypo-LL SI) / (Liver SI + Hypo-LL SI)

- Hyper-LTLC= (Hyper-LL SI - Liver SI) / (Liver SI + Hyper-LL SI)

\section{Statistical analysis}

Subjective evaluations were compared using paired Wilcoxon tests and the inter-reader reliability was analyzed using weighted kappa coefficients. Bivariate correlation analyses were performed using either Pearson's r or Spearman's rho. RLSI, LTVC, Hypo-LTLC, and Hyper-LTLC were compared between Cartesian-eTHRIVE and Radial-eTHRIVE using paired t-tests. Comparisons are two-sided and considered statistically significant at $p<0.05$.

\section{Results}

The mean patient age was $60.4 \pm 12.1$ years; 48 men (55.8\%) and 38 women (44.2\%). Cartesian-eTHRIVE and Radial-eTHRIVE showed no significant difference with respect to the time interval after contrast administration with $11.7 \pm 2.0$ min versus $11.2 \pm 2.2$ $\min ; \mathrm{p}=0.126$.

\section{Subjective analysis}

Both readers scored a higher anatomic and vessel sharpness for the Radial-eTHRIVE sequence compared to the Cartesian-eTHRIVE sequence (anatomic sharpness: $1.29 \pm 0.53$ vs. $1.53 \pm 0.75$; $p=0.006$ and $1.33 \pm 0.52$ vs. $1.56 \pm 0.83 ; p=0.009$; vessel sharpness: $1.33 \pm 0.62$ vs. $1.74 \pm 0.90 ; p=0.000$ and $1.31 \pm 0.54$ vs. 1.56 $\pm 0.82 ; p=0.012$ ) ( $\triangleright$ Fig. 2 ). Reader 2 evaluated diagnostic quality to be significantly better in the Radial-eTHRIVE with $1.36 \pm 0.55$ vs. $1.58 \pm 0.99 ; p=0.030$, whereas reader 1 did not assess a significant difference concerning diagnostic quality over all patients. Reader 1 rated the image contrast to be significantly better in the CartesianeTHRIVE sequence with $1.33 \pm 0.56$ vs. $1.52 \pm 0.65 ; p=0.012$, while the ratings by reader 2 were not statistically significant. The detailed subjective results are shown in $>$ Table 3.

\section{Objective analysis}

Objective measurements revealed no statistically significant difference between Cartesian-eTHRIVE and Radial-eTHRIVE concerning RLSI and LTVC. A higher lesion-to-liver contrast for hypointense focal liver lesions (Hypo-LTLC) was observed for Cartesian-eTHRIVE with $0.33 \pm 0.19$ vs. $0.20 \pm 0.11 ; p=0.000$ ( Fig. 3 ), while no difference in lesion-to-liver contrast was measured for hyperintense liver lesions (Hyper-LTLC) with $0.14 \pm 0.08$ vs. $0.14 \pm 0.09 ; p=0.982$. The detailed objective results are shown in $>$ Table 4.

\section{Subgroup analysis}

The subgroup of patients with breathing artifacts (artifact score $\geq 3$ ) based on conventional breath-hold Cartesian-eTHRIVE comprised 9 out of the total 86 patients (representing $10.5 \%$ of the whole study population) and were all identified by both readers independently. In this subgroup analysis, Radial-eTHRIVE yielded better results in all five subjective categories which reached statistical significance for both readers and all categories. Radial-eTHRIVE images in this subgroup still showed a "very good" (Reader 1: $55.6 \%$, Reader 2: $44.4 \%)$, "good" (Reader 1: 22.2\%, Reader 2: 44.4\%) or "fair" (Reader 1: $22.2 \%$, Reader 2: $11.1 \%$ ) overall diagnostic quality ( $>$ Fig. 4 ). Detailed subgroup results are shown in $\triangleright$ Table 5 . 
- Table 2 Likert scale ratings for assessed aspects of subjective image quality.

- Tab.2 Likert-Skala-Bewertungen für die untersuchten Aspekte der subjektiven Bildqualität.

\begin{tabular}{|c|c|c|}
\hline $\begin{array}{l}\text { image quality } \\
\text { parameter }\end{array}$ & score & scoring system \\
\hline artifact impact & $1-5$ & $\begin{array}{l}\text { - } 1: \text { not present } \\
\text { - } 2 \text { : low impact } \\
\text {. } 3 \text { : moderate impact } \\
\text { - } 4 \text { : strong impact } \\
\text { - } 5 \text { : non-diagnostic }\end{array}$ \\
\hline anatomic sharpness & $1-5$ & $\begin{array}{l}\text { - } 1: \text { very good } \\
\text { - } 2: \text { good } \\
\text { - } 3: \text { fair } \\
\text { - } 4 \text { : poor } \\
\text { - } 5: \text { non-diagnostic }\end{array}$ \\
\hline vessel sharpness & $1-5$ & $\begin{array}{l}\text { - } 1: \text { very good } \\
\text { - } 2: \text { good } \\
\text { - } 3: \text { fair } \\
\text { - } 4: \text { poor } \\
\text { - } 5: \text { non-diagnostic }\end{array}$ \\
\hline diagnostic quality & $1-5$ & $\begin{array}{l}\text { - } 1 \text { : very good } \\
\text { - } 2 \text { good } \\
\text { - } 3 \text { : fair } \\
\text { - } 4 \text { : poor } \\
\text { - } 5 \text { : non-diagnostic }\end{array}$ \\
\hline contrast & $1-5$ & $\begin{array}{l}\text { - } 1 \text { : very good } \\
\text { - } 2 \text { : good } \\
\text { - } 3 \text { : fair } \\
\text { - } 4: \text { poor } \\
\text { - } 5 \text { : non-diagnostic }\end{array}$ \\
\hline
\end{tabular}

\section{Inter-reader reliability and inter-reader agreement}

Inter-reader reliability analysis showed good concordance with an overall weighted Cohen's kappa of 0.694 . Weighted Cohen's kappa values demonstrated good concordance for all of the subgroups: "artifact rating" with 0.703 , "anatomic sharpness" with 0.651 , "vessel sharpness" with 0.622 , "diagnostic quality" with 0.780 , and "image contrast" with $0.713(\triangleright$ Table 6$)$.

\section{Discussion}

The contrast-enhanced T1-weighted fat-saturated sequence ( $\mathrm{T} 1 \mathrm{w}$-FS) is one of the most important sequences in state-of-theart liver MR protocols and is typically acquired as dynamic contrast-enhanced (DCE-) MRI with subsequent breath-holds of about $15-20$ seconds during arterial to delayed phases [21]. Based on the literature, about $10-12 \%$ of patients have difficulty holding their breath after injection of extracellular Gd-based contrast agents $[22,23]$ and even higher rates of up to $18 \%$ of transient motion artifacts during arterial phase T1w-FS imaging after injection of hepatocyte-specific Gd-EOB-DTPA have been reported [23]. Breathing artifacts can dramatically impact image quality, potentially leading to a non-diagnostic MR examination. In our

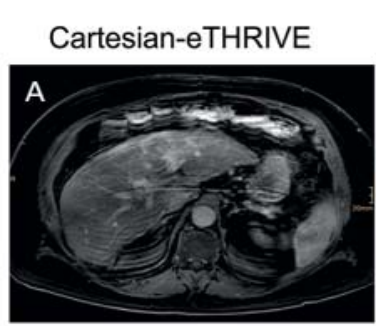

\section{Radial-eTHRIVE}
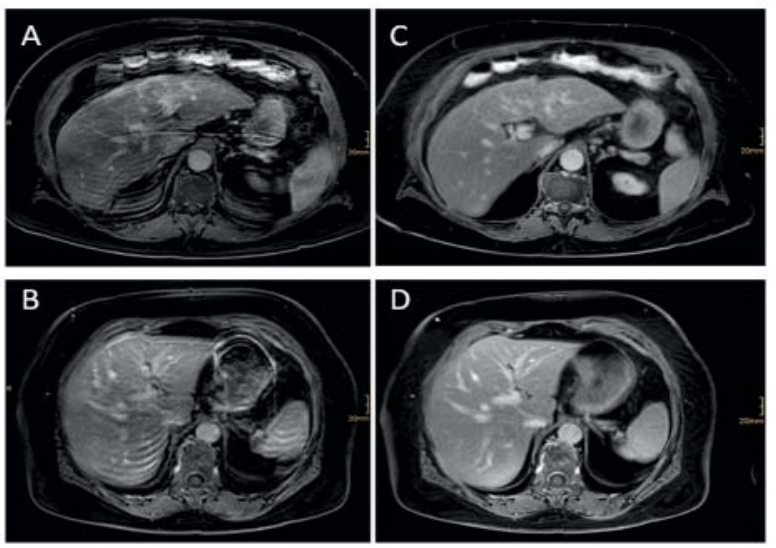

- Fig. 2 Cartesian-eTHRIVE (A, B) and Radial-eTHRIVE (C, D) of two exemplary patients (A/C and B/D). Breath-hold Cartesian-eTHRIVE images with impaired image quality due to motion artifacts (A, B) and free-breathing Radial-eTHRIVE counterparts with good image quality and only few streaking artifacts (C, D).

- Abb. 2 Breath-hold-Cartesian-eTHRIVE (A, B ) von zwei Patienten (A/C, B/D) mit Atemartefakten und die korrespondierende RadialeTHRIVE in freier Atmung (C, D), die eine weiterhin gute Bildqualität zeigen und lediglich geringe Streifenartefakte aufweisen.
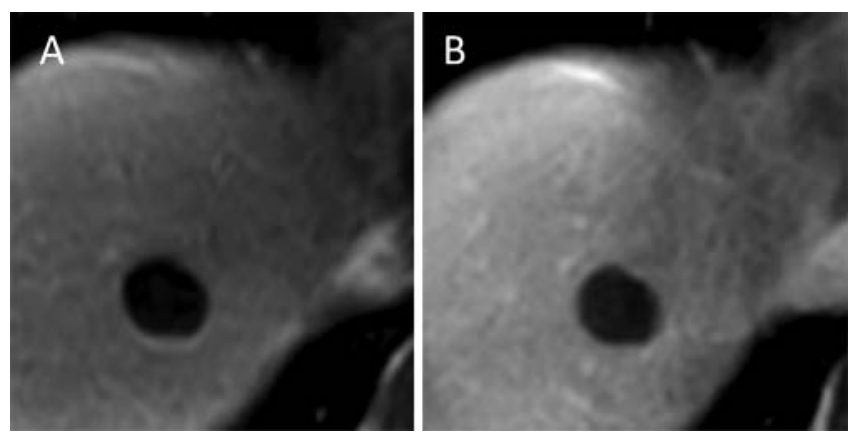

- Fig. 3 Compared to Cartesian-eTHRIVE A, increased signal intensity of hypointense liver cyst in Radial-eTHRIVE B.

- Abb. 3 Im Vergleich zur Cartesian-eTHRIVE A zeigt B eine gering angehobene Signalintensität in einer hypointensen Leberzyste in der Radial-eTHRIVE.

study, $10.5 \%$ of patients demonstrated relevant breathing artifacts during conventional Cartesian-eTHRIVE in delayed phase imaging which is in line with the published literature. Dynamic contrast-enhanced (DCE) arterial and portal phases are important for the detection and characterization of focal liver lesions. However, delayed contrast phase imaging is a crucial part of MR protocols using both extracellular contrast agents, e. g. for detection of hypoperfused liver metastasis and visualization of intralesional "wash-out" of hepatocellular carcinoma, and hepatocyte-specific MRI, e. g. for detection of small liver metastases $(<10 \mathrm{~mm})[24-26]$. In the current study, measurements were performed after about 10 min delay following contrast adminis- 
- Table 3 Objective characteristics of Cartesian-eTHRIVE and Radial-eTHRIVE.

- Tab.3 Objektive Charakteristika der Cartesian-eTHRIVE und der Radial-eTHRIVE.

\begin{tabular}{|l|l|l|l|}
\hline & n & Cartesian-eTHRIVE & Radial-eTHRIVE \\
\hline relative liver signal intensity (RLSI) & 86 & $1.073 \pm 0.113$ & $1.078 \pm 0.124$ \\
\hline liver-to-vessel contrast (LTVC) & 86 & $0.118 \pm 0.102$ & $0.126 \pm 0.097$ \\
\hline hypointense lesion-to-liver contrast (HLTLC) & 42 & $0.325 \pm 0.194$ & $0.203 \pm 0.108$ \\
\hline hyperintense lesion-to-liver contrast (HLTLC) & 8 & $0.142 \pm 0.083$ & $0.143 \pm 0.088$ \\
\hline time after i. v. contrast [min] & 86 & $11.7 \pm 2.0$ & $11.2 \pm 2.2$ \\
\hline
\end{tabular}

- Table 4 Results of subjective image quality ratings by Reader 1 and 2 . Statistically significant results are marked in bold $(p<0.05)$. Values are given as mean \pm standard deviation.

- Tab.4 Ergebnisse der subjektiven Bewertungen der Bildqualität durch Reader 1 und Reader 2. Statistisch signifikante Ergebnisse sind fett markiert $(p<0.05)$. Die Werte sind als Mittelwert \pm Standardabweichung angegeben.

\begin{tabular}{|c|c|c|c|c|c|c|}
\hline & \multicolumn{3}{|l|}{ reader 1} & \multicolumn{3}{|l|}{ reader 2} \\
\hline & Cartesian-eTHRIVE & Radial-eTHRIVE & $\mathbf{P}$ & Cartesian-eTHRIVE & Radial-eTHRIVE & $\mathbf{P}$ \\
\hline artifact impact & $1.57 \pm 0.84$ & $1.42 \pm 0.60$ & 0.139 & $1.55 \pm 0.89$ & $1.50 \pm 0.55$ & 0.653 \\
\hline anatomic sharpness & $1.53 \pm 0.78$ & $1.29 \pm 0.53$ & 0.006 & $1.56 \pm 0.83$ & $1.33 \pm 0.52$ & 0.009 \\
\hline vessel sharpness & $1.74 \pm 0.90$ & $1.33 \pm 0.62$ & 0.000 & $1.56 \pm 0.82$ & $1.31 \pm 0.54$ & 0.012 \\
\hline diagnostic quality & $1.50 \pm 0.89$ & $1.40 \pm 0.64$ & 0.288 & $1.58 \pm 0.99$ & $1.36 \pm 0.55$ & 0.030 \\
\hline contrast & $1.33 \pm 0.56$ & $1.52 \pm 0.65$ & 0.012 & $1.33 \pm 0.52$ & $1.36 \pm 0.51$ & 0.567 \\
\hline
\end{tabular}

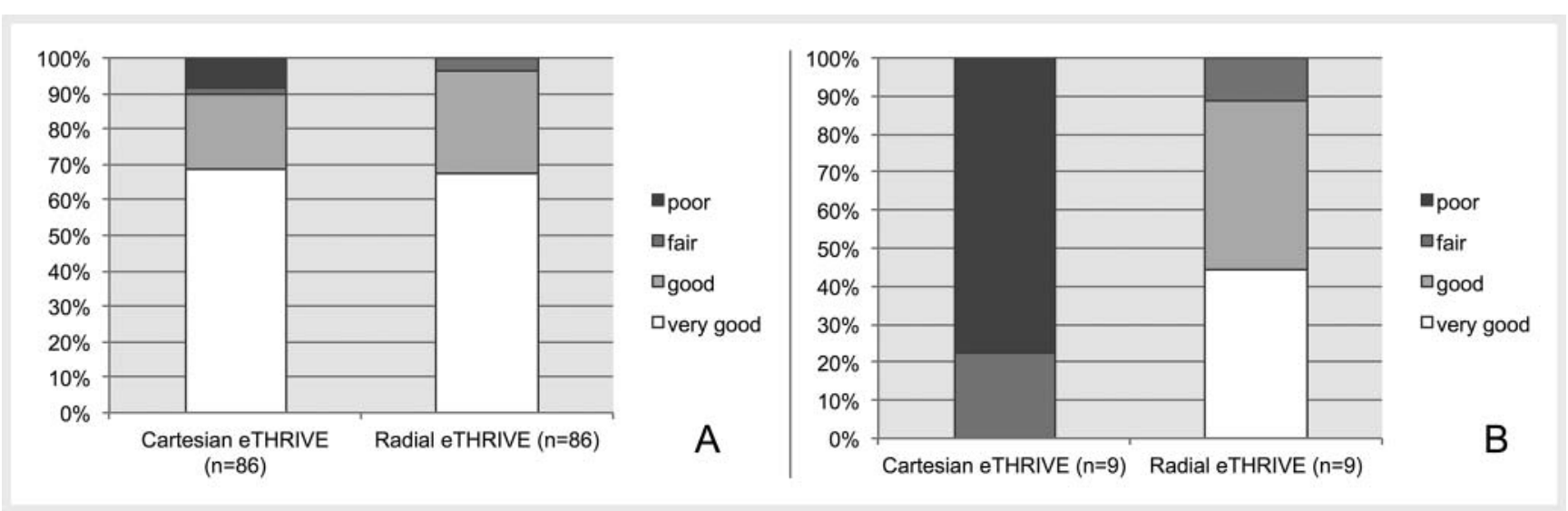

- Fig. 4 Percentages of subjective "diagnostic quality“ ratings for all subjects A, and percentages of „diagnostic quality“ ratings for a subgroup of patients with impaired breath-holding capability B, defined as artifact impact score $\geq 3$ in conventional Cartesian-eTHRIVE (Reader 1 ).

- Abb.4 Anteil der subjektiven Bewertungen der diagnostischen Bildqualität in der Cartesian-eTHRIVE und Radial-eTHRIVE für alle Patienten A sowie für die Subgruppe der Patienten mit ausgeprägten Atemartefakten in der konventionellen Cartesian-eTHRIVE B.

tration in order to allow stable contrast conditions for both MR scans.

In our study, conventional breath-hold Cartesian-eTHRIVE demonstrated a similar or slightly inferior subjective image quality in comparison to free-breathing Radial-eTHRIVE over all evaluated
MR examinations. This observation is in accordance with previous studies which found similar results with a free-breathing 3 D T1-FS FFE sequence with radial k-space sampling [15, 27 - 30]. However, these studies only investigated the free-breathing techniques in comparatively small cohorts of patients. In contrast to the pub- 
- Table 5 Results of subjective image quality ratings by Reader 1 and 2 for a subgroup of patients with insufficient breath-hold. Statistically significant results are marked in bold $(p<0.05)$. Values are given as mean \pm standard deviation.

- Tab.5 Ergebnisse der subjektiven Bewertungen der Bildqualität durch Reader 1 und Reader 2 für eine Subgruppe von Patienten mit starken Atemartefakten in der konventionellen Cartesian-eTHRIVE. Statistisch signifikante Ergebnisse sind fett markiert $(p<0.05)$. Die Werte sind als Mittelwert \pm Standardabweichung angegeben.

\begin{tabular}{|c|c|c|c|c|c|c|}
\hline & \multicolumn{3}{|l|}{ reader $1(n=9)$} & \multicolumn{3}{|l|}{ reader $2(n=9)$} \\
\hline & Cartesian-eTHRIVE & Radial-eTHRIVE & $\mathbf{p}$ & Cartesian-eTHRIVE & Radial-eTHRIVE & $\mathbf{p}$ \\
\hline artifact impact & $3.6 \pm 0.53$ & $1.6 \pm 0.88$ & 0.000 & $3.7 \pm 0.87$ & $1.7 \pm 0.87$ & 0.003 \\
\hline anatomic sharpness & $3.2 \pm 0.67$ & $1.3 \pm 0.71$ & 0.000 & $3.4 \pm 0.88$ & $1.4 \pm 0.73$ & 0.000 \\
\hline vessel sharpness & $3.6 \pm 0.73$ & $1.6 \pm 0.88$ & 0.000 & $3.3 \pm 1.0$ & $1.4 \pm 0.73$ & 0.003 \\
\hline diagnostic quality & $3.8 \pm 0.44$ & $1.7 \pm 0.87$ & 0.000 & $4.1 \pm 0.6$ & $1.7 \pm 0.7$ & 0.000 \\
\hline contrast & $2.1 \pm 0.60$ & $1.4 \pm 0.53$ & 0.022 & $2.1 \pm 0.6$ & $1.4 \pm 0.53$ & 0.022 \\
\hline
\end{tabular}

- Table 6 Weighted-Cohen's-kappa-values for interreader reliability of subjective ratings.

- Tab. 6 Gewichtete Cohen's-kappa-Analyse als Maß für die Interreader-Reliabilität.

interreader reliability

\begin{tabular}{|l|l|}
\hline & weighted-Cohen's-kappa \\
\hline artifact impact & 0.703 \\
\hline anatomic sharpness & 0.650 \\
\hline vessel sharpness & 0.622 \\
\hline diagnostic quality & 0.780 \\
\hline contrast & 0.713 \\
\hline
\end{tabular}

lished studies, our free-breathing Radial-eTHRIVE approach combines radial k-space sampling with "pseudo golden angle" sampling in the axial plane, Cartesian encoding in the z-direction, SENSE parallel imaging in the z-direction, and partial Fourier techniques ( $\triangleright$ Fig. 1 ). The standard uniform (non-golden angle) ordering scheme, as described by Chandarana et al. [15], is known to be more susceptible to streaking artifacts caused by motion due to their equidistant k-space nature. The proposed "pseudo golden angle" sampling scheme used in our study leads to a perfectly distributed k-space while assuring a low correlation of variations caused by motion within the k-space. This results in reduced streaking artifacts compared to standard non-uniform sampling schemas, as described by Winkelmann et al. [31]. Our investigated Radial-eTHRIVE sequence demonstrated superior subjective image ratings with respect to anatomical and vessel sharpness. This can probably be ascribed to an increased robustness against patient motion of Radial-eTHRIVE compared to Cartesian-eTHRIVE due to the intrinsic oversampling of the k-space center in combination with the pseudo golden angle increment. The subjective and objective analysis of image contrast showed that free-breathing Radial-eTHRIVE had inferior performance compar- ed to conventional breath-hold Cartesian-eTHRIVE. The objective lesion-to-liver contrast ratio of hypointense liver lesions (HypoLTLC) was significantly lower in Radial-eTHRIVE images. This might be caused by residual motion in the raw data used for reconstruction, resulting in "signal smearing" between different anatomical structures with different signal intensities ( $>$ Fig. 3). This effect was substantially lower for signal intensity measurements of hyperintense liver lesions (Hyper-LTLC), which might be caused by the lower intrinsic contrast of hyperperfused liver lesions in the delayed imaging phase. However, the limited number of hyperintense liver lesions in the delayed contrast phase in our study hampers a final appraisal.

Most importantly, in the subgroup analysis of patients who showed motion artifacts with a reduction in image quality in the breath-hold Cartesian-eTHRIVE sequence (artifact score $\geq 3$ ), the free-breathing Radial-eTHRIVE sequence still showed highly diagnostic images and was superior in every rated subjective imaging category ("artifact impact", "anatomic sharpness", "vessel sharpness", "contrast impression", and "overall diagnostic quality") compared to Cartesian-eTHRIVE. This represents a valuable advantage of Radial-eTHRIVE for liver MRI in clinical practice for patients who are unable to suspend respiration adequately. In contrast to other motion artifact reduction techniques, such as respiratory-gated image acquisition, no further hardware, like respiration belts, and no complex post-processing methods are needed for Radial-eTHRIVE. Moreover, the total scan time of Radial-eTHRIVE does not depend on breathing pattern or gating efficiencies. Thus, free-breathing Radial-eTHRIVE sequences are very easy to add to an imaging protocol if standard breath-hold Cartesian-eTHRIVE does not reach sufficient quality due to motion-induced artifacts.

However, due to the comparably long scan time of RadialeTHRIVE, as presented in this study, it is currently not suitable to fully apply this technique in DCE MRI. However, new developments to overcome this limitation by using parallel imaging and compressed sensing-based image reconstruction have been suggested and demonstrated [32]. These techniques might also be used to improve image contrast and address remaining image 
degradation due to residual respiratory motion artifacts in RadialeTHRIVE. Obviously, careful clinical evaluation of these new techniques is needed in future studies.

Most artifacts observed in the Radial-eTHRIVE images were streaking artifacts which are known to occur with radial k-space sampling and are either caused by k-space undersampling or by patient motion. In the current study no undersampling was used in the radial direction, thus the observed streaking artifacts can most likely be ascribed to patient motion. Since the current data showed a very heterogeneous impact of streaking artifacts, we hypothesize that deep and/or irregular breathing patterns are associated with stronger streaking artifacts as compared to shallow and regular respiration. Although patients were instructed to maintain shallow and regular breathing during the free-breathing Radial-eTHRIVE sequence, a limitation of the present study is that this was not controlled and documented. Furthermore, we did not systematically compare different radial k-space sampling regimes. However, this was beyond the scope of the current study and future studies evaluating the presented "pseudo golden angle" Radial-eTHRIVE with respiratory gating and tracking techniques are warranted. A combined technique might further reduce the effect of breathing variabilities - like deep and irregular breathing patterns. While a total of 86 patients were included in our study (as compared to 18 patients in Chandarana et al. [15]), the interesting observation of the robustness of Radial-eTHRIVE is based on 9 patients with motion artifacts in conventional CartesianeTHRIVE. The small patient number of this subgroup has to be addressed as a limitation, but only about $10 \%$ of the patients demonstrated relevant breathing artifacts, which is in line with the prevalence of breathing-related motion-induced artifacts of $10-12 \%$ after injection of extracellular Gd-based contrast agents $[22,23]$.

In conclusion, the free-breathing Radial-eTHRIVE sequence is a valid tool for delayed-phase imaging of the liver providing diagnostic image quality even in patients who cannot adequately hold their breath. Despite remaining shortcomings, such as longer scan times and lower contrast, free-breathing Radial-eTHRIVE with "pseudo golden angle" k-space sampling represents a valuable and robust add-on sequence in patients with impaired respiration.

\section{CLINICAL RELEVANCE}

In liver MRI, free-breathing contrast-enhanced radial T1w-FSFFE (Radial-eTHRIVE) as an add-on sequence can yield high diagnostic image quality and provide helpful diagnostic information in patients with significant breathing artifacts and limited image quality.

\begin{tabular}{|c|c|}
\hline \multicolumn{2}{|l|}{ ABBREVIATIONS } \\
\hline T1w & T1-weighted \\
\hline FB & free-breathing \\
\hline FS & fat suppression \\
\hline FFE & fast field echo \\
\hline MRI & magnetic resonance imaging \\
\hline eTHRIVE & $\begin{array}{l}\text { enhanced T1 high-resolution } \\
\text { isotropic volume excitation }\end{array}$ \\
\hline Cartesian-eTHRIVE & $\begin{array}{l}\text { eTHRIVE with Cartesian k-space } \\
\text { sampling }\end{array}$ \\
\hline Radial-eTHRIVE & $\begin{array}{l}\text { eTHRIVE with pseudo golden angle } \\
\text { radial k-space sampling }\end{array}$ \\
\hline FLL & focal liver lesions \\
\hline $3 \mathrm{D}$ & 3-dimensional \\
\hline DCE & dynamic contrast-enhanced \\
\hline $\mathrm{BH}$ & breath-hold \\
\hline ROI & region-of-interest \\
\hline SI & signal intensity \\
\hline PVSI & portal venous signal intensity \\
\hline Hypo-LL & hypointense liver lesion \\
\hline Hyper-LL & hyperintense liver lesion \\
\hline RLSI & relative liver-signal-intensity ratio \\
\hline LTVC & liver-to-vessel contrast ratio \\
\hline Hypo-LTLC & $\begin{array}{l}\text { hypointense lesion-to-liver contrast } \\
\text { ratio }\end{array}$ \\
\hline Hyper-LTLC & $\begin{array}{l}\text { hyperintense-lesion-to-liver-con- } \\
\text { trast ratio }\end{array}$ \\
\hline
\end{tabular}

Conflict of Interest

KW und GB are staff members of Philips. Both authors had no influence on the evaluation of the clinical data. The other authors declare that they have no conflict of interest.

\section{References}

[1] Guang Y et al. Diagnosis value of focal liver lesions with SonoVue(R)-enhanced ultrasound compared with contrast-enhanced computed tomography and contrast-enhanced MRI: a meta-analysis. J Cancer Res Clin Oncol 2011; 137: 1595-1605

[2] Eiber $\mathrm{M}$ et al. Detection and classification of focal liver lesions in patients with colorectal cancer: retrospective comparison of diffusion-weighted MR imaging and multi-slice CT. Eur J Radiol 2012; 81: 683-691

[3] Banerjee $R$ et al. Multiparametric magnetic resonance for the non-invasive diagnosis of liver disease. J Hepatol 2014; 60: 69-77

[4] Sommer WH et al. Contrast agents as a biological marker in magnetic resonance imaging of the liver: conventional and new approaches. Abdom Imaging 2012; 37: 164-179

[5] Grazioli L et al. Hepatocellular adenoma and focal nodular hyperplasia: value of gadoxetic acid-enhanced MR imaging in differential diagnosis. Radiology 2012; 262: 520-529

[6] Hedderich DM et al. Modern magnetic resonance imaging of the liver. Radiologe 2015; 55: 1045-1056

[7] ACR. ACR-SAR-SPR PRACTICE PARAMETER FOR THE PERFORMANCE OF MAGNETIC RESONANCE IMAGING (MRI) OF THE LIVER. 2015. [cited 
2016 03/15], Available from: http://www.acr.org/ /media/ACR/Documents/PGTS/guidelines/MRI_Liver.pdf

[8] Sirlin CB et al. Consensus report from the 6th International forum for liver MRI using gadoxetic acid. J Magn Reson Imaging 2014; 40: 516-529

[9] Luetkens JA et al. Respiratory motion artefacts in dynamic liver MRI: a comparison using gadoxetate disodium and gadobutrol. Eur Radiol 2015; 25: $3207-3213$

[10] Inoue $Y$ et al. Optimal techniques for magnetic resonance imaging of the liver using a respiratory navigator-gated three-dimensional spoiled gradient-recalled echo sequence. Magn Reson Imaging 2014; 32: 975 - 980

[11] Ogasawara G et al. Evaluation of a respiratory navigator-gating technique in Gd-EOB-DTPA-enhanced magnetic resonance imaging for the assessment of liver tumors. Eur J Radiol 2016; 85: 1232 - 1237

[12] Vasanawala SS et al. Navigated abdominal T1-W MRI permits freebreathing image acquisition with less motion artifact. Pediatr Radiol 2010; 40: $340-344$

[13] Pruessmann KP et al. SENSE: sensitivity encoding for fast MRI. Magn Reson Med 1999; 42: 952 - 962

[14] Griswold MA et al. Generalized autocalibrating partially parallel acquisitions (GRAPPA). Magn Reson Med 2002; 47: 1202 - 1210

[15] Chandarana $\mathrm{H}$ et al. Free-breathing radial 3D fat-suppressed T1-weighted gradient echo sequence: a viable alternative for contrast-enhanced liver imaging in patients unable to suspend respiration. Invest Radiol 2011; 46: 648-653

[16] Reiner CS et al. Contrast-enhanced free-breathing 3D T1-weighted gradient-echo sequence for hepatobiliary MRI in patients with breath-holding difficulties. European Radiology 2013; 23: 3087-3093

[17] Chandarana $\mathrm{H}$ et al. Free-Breathing Contrast-Enhanced Multiphase MRI of the Liver Using a Combination of Compressed Sensing, Parallel Imaging, and Golden-Angle Radial Sampling. Invest Radiol 2013; 48: 10 - 16

[18] Davenport MS et al. Comparison of acute transient dyspnea after intravenous administration of gadoxetate disodium and gadobenate dimeglumine: effect on arterial phase image quality. Radiology 2013; 266 : $452-461$

[19] Lee MS et al. Gadoxetic acid disodium-enhanced magnetic resonance imaging for biliary and vascular evaluations in preoperative living liver donors: comparison with gadobenate dimeglumine-enhanced MRI. J Magn Reson Imaging 2011; 33: 149-159
[20] Fenzi A, Bortolazzi M, Marzola P. Comparison between signal-to-noise ratio, liver-to-muscle ratio, and 1/T2 for the noninvasive assessment of liver iron content by MRI. J Magn Reson Imaging 2003; 17: 589-592

[21] Saito K et al. Assessing liver function using dynamic Gd-EOB-DTPA-enhanced MRI with a standard 5-phase imaging protocol. J Magn Reson Imaging 2013; 37: 1109-1114

[22] Motosugi U et al. An Investigation of Transient Severe Motion Related to Gadoxetic Acid-enhanced MR Imaging. Radiology 2016; 279: 93 - 102

[23] Davenport MS et al. Matched within-patient cohort study of transient arterial phase respiratory motion-related artifact in MR imaging of the liver: gadoxetate disodium versus gadobenate dimeglumine. Radiology 2014; $272: 123-131$

[24] Huppertz A et al. Improved detection of focal liver lesions at MR imaging: multicenter comparison of gadoxetic acid-enhanced MR images with intraoperative findings. Radiology 2004; 230: 266-275

[25] Motosugi $U$ et al. Detection of pancreatic carcinoma and liver metastases with gadoxetic acid-enhanced MR imaging: comparison with contrast-enhanced multi-detector row CT. Radiology 2011; 260: 446-453

[26] Lee Y] et al. Hepatocellular carcinoma: diagnostic performance of multidetector CT and MR imaging-a systematic review and meta-analysis. Radiology 2015; 275: $97-109$

[27] Chandarana $\mathrm{H}$ et al. Free-breathing contrast-enhanced $\mathrm{T} 1$-weighted gradient-echo imaging with radial k-space sampling for paediatric abdominopelvic MRI. Eur Radiol 2014; 24: 320-326

[28] Shin $\mathrm{H}$ ] et al. Comparison of image quality between conventional VIBE and radial VIBE in free-breathing paediatric abdominal MRI. Clin Radiol 2016; 71: $1044-1049$

[29] Budjan J et al. Rapid Cartesian versus radial acquisition: comparison of two sequences for hepatobiliary phase MRI at 3 tesla in patients with impaired breath-hold capabilities. BMC Med Imaging 2017; 17: 32

[30] Reiner CS et al. Contrast-enhanced free-breathing 3D T1-weighted gradient-echo sequence for hepatobiliary MRI in patients with breath-holding difficulties. Eur Radiol 2013; 23: 3087 - 3093

[31] Winkelmann S et al. An optimal radial profile order based on the Golden Ratio for time-resolved MRI. IEEE Trans Med Imaging 2007; 26: 68 - 76

[32] Chandarana H et al. Respiratory Motion-Resolved Compressed Sensing Reconstruction of Free-Breathing Radial Acquisition for Dynamic Liver Magnetic Resonance Imaging. Invest Radiol 2015; 50: 749-756 